\title{
Leonardo da Vinci's The Last Supper: Reconstruction of the Room Using Reverse Geometric Perspective Processes
}

\author{
N. Kourniatis*, N. T. U. A. Architect \\ Department of Civil Engineering, University of West Attica, Athens, Greece \\ Email: Kourniatis_geometry@uniwa.gr
}

How to cite this paper: Kourniatis, N. and Architect, N.T.U.A. (2019) Leonardo da Vinci's The Last Supper. Reconstruction of the Room Using Reverse Geometric Perspective Processes. Journal of Applied Mathematics and Physics, 7, 1941-1957. https://doi.org/10.4236/jamp.2019.79134

Received: July 22, 2019

Accepted: August 31, 2019

Published: September 3, 2019

Copyright () 2019 by author(s) and Scientific Research Publishing Inc. This work is licensed under the Creative Commons Attribution International License (CC BY 4.0). http://creativecommons.org/licenses/by/4.0/

\begin{abstract}
This article presents a geometric exploration of the reconstruction of a model of the three-dimensional space that Leonardo da Vinci had before him or imagined when he created The Last Supper. The purpose is to reveal the geometrical principles inherent in this work of art, as well as to propose an invert geometrical method, from 2D to 3D space reconstruction.
\end{abstract}

\section{Keywords}

Perspective, Geometrical Reconstruction, The Last Supper, Leonardo da Vinci

\section{Introduction}

This exploration begins by describing the method used to construct a perspective drawing, in which the role of three elements is highlighted: the base line of the picture plane, on which the perspective drawing is made, the height of the horizon and how it is depicted on the picture plane-which is connected to the height of the artist's station point and, generally speaking, to the work of art's viewing height - and, lastly, the distance from the picture plane at which the artist positions the viewer in order to better observe the work of art. These three concepts, namely the picture plane's base line, horizon and viewing distance, play a decisive role in the work of art. Once the geometric meanings of these concepts have been presented, using reverse geometric perspective processes we conduct a geometric abstraction on da Vinci's painting in order to determine its base line, horizon line and the viewing distance the artist has set. The article ends with a presentation of the three-dimensional space model resulting from the preceding

${ }^{*}$ Assist. Prof. at UNIWA. 
process and an analysis of whether the perspective image of the model, from the viewpoint set by the artist, coincides with the actual work of art.

\section{Geometric Interpretation of Base Line, Horizon Line and Viewing Distance in a Perspective Drawing}

A perspective drawing is the central projection of an object or space on a plane (Figure 1) [1]. When we talk about the centre of projection, we mean the observer's viewpoint, whereas central projection refers to where the visual rays intersect the aforementioned plane. Visual rays emanate from the centre of projection and end at all the key points of an object, intersecting the plane on the way. The object's perspective image, namely its central projection from the particular centre of projection, is created on this plane. The intersected plane is called the picture plane and is usually vertical, in other words perpendicular to the ground plane on which the viewer stands. The vertical picture plane meets the ground plane along a straight line called the base line. When we speak about the distance between the centre of projection, from now on referred to as the station point or viewpoint, and the picture plane, we mean how far away the viewer (or artist) stands from the plane. Up to now, we have defined the base line of a picture plane and the viewer's distance from this plane. Next we will explain the meaning of "horizon line".

\subsection{Perspective of a Horizontal Line and the Horizon Line}

To define the horizon line [1], we ought to mention the existence, in the construction of a perspective image, of a horizontal line belonging on the horizontal plane on which the observer stands (Figure 2). As mentioned before, the perspective image of an object on a plane known as a picture plane is the intersection of the visual rays, which connect the viewpoint and the object, with this plane. To determine the perspective of a straight line on the picture plane, which will also be a straight line, all we have to do is determine the perspectives of two of its points. In Figure 2 we observe that the visual ray corresponding to point 1

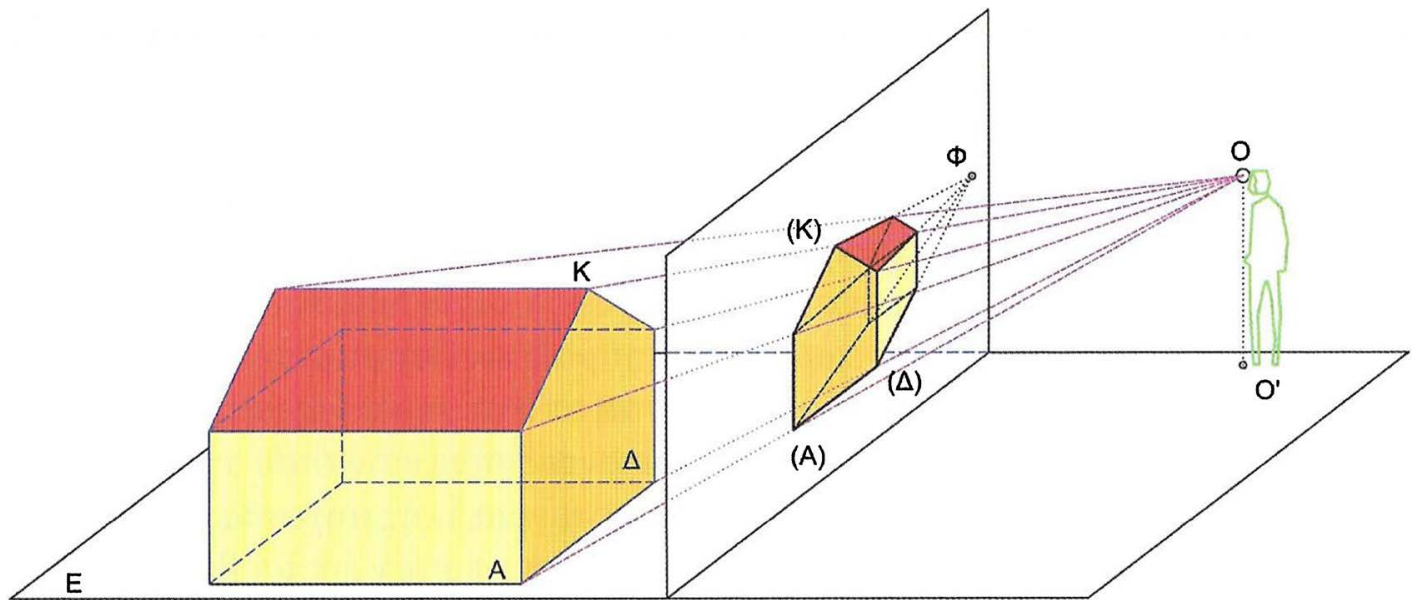

Figure 1. The projection. 


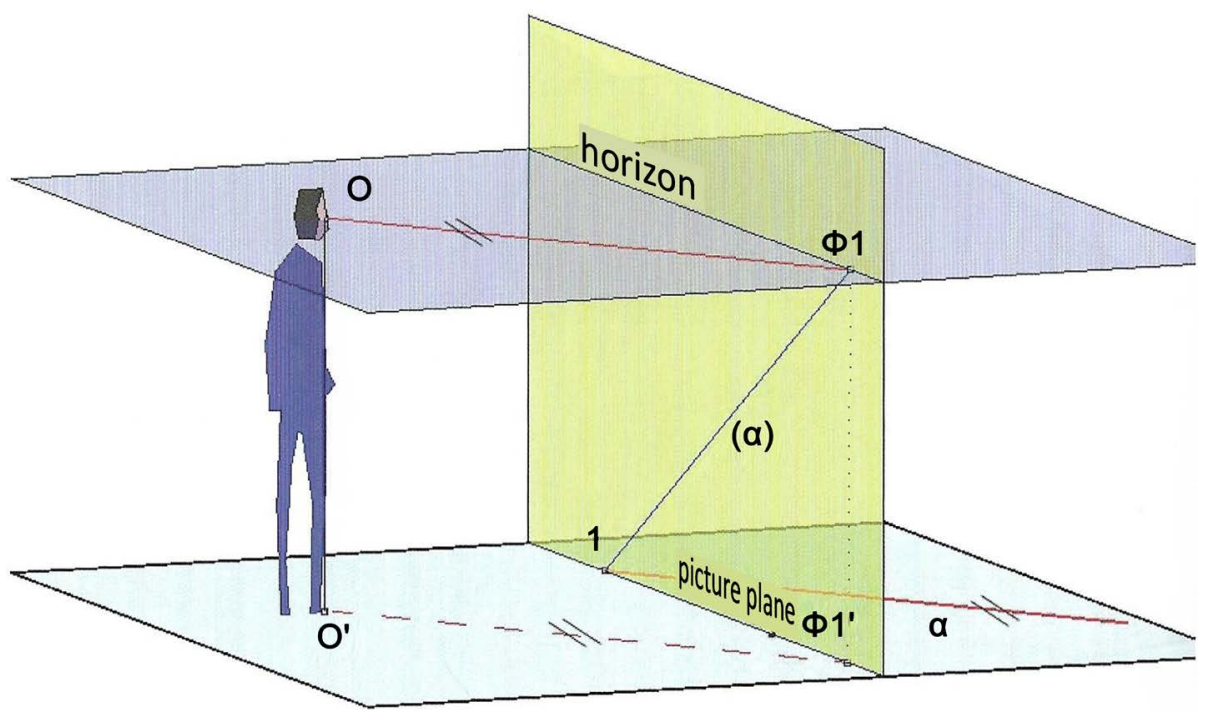

Figure 2. The perspective of a horizontal line.

on horizontal line $\alpha$ also meets the picture plane at 1 , since that is already on the picture plane. Thus, point 1's perspective will be itself. We therefore need the perspective of one more point to determine the perspective of straight line $\alpha$. Let us say that we choose to construct the perspective of a point on $\alpha$ that is an infinite distance away from the viewer, in other words, $\alpha$ 's point at infinity. For this purpose, let us consider the visual ray that connects the observer's viewpoint with the point at infinity. This means that the ray will be parallel to $\alpha$ and will intersect the picture plane at a point we refer to as $\alpha$ 's vanishing point $\Phi 1$. Line $\alpha$ 's vanishing point is the perspective of the point along its length that is an infinite distance away from the viewer. If we then join $\Phi 1$ with 1, we will have the perspective image on the picture plane of $\alpha$ from 1 to infinity. Let us now consider the horizontal plane that is at the observer's eye level. The line formed where this plane intersects the picture plane is called the horizon line. All the vanishing points of the horizontal lines in physical space are located along this line. In classical perspective drawing, horizontal lines have vanishing points along the horizon line, and straight lines that are parallel in space have the same vanishing point in perspective. This is what happens in physical space. In drawing, in other words on paper, we consider the picture plane to have been rotated on its base and collapsed on the horizontal plane. Thus, on paper we have the base line, the horizon line and the projection of station point $\mathrm{O}^{\prime}$. On paper we do not have the actual viewpoint, that is, $\mathrm{O}$. It is, however, important to remember that the distance between the horizon line and the base line represents the viewing height and the distance between $\mathrm{O}^{\prime}$ and the base line represents how far the observer is standing from the painting.

\subsection{The Geometric Concept of the Visual Ray}

Figure 3 shows us the perspective of a point A along the straight line $\alpha$. Visual ray OA meets the picture plane at point $(\mathrm{A})$, which can also be located as follows 
[1]: The right-angled triangle $\mathrm{OO}^{\prime} \mathrm{A}$ is created in space, with a right angle at $\mathrm{O}^{\prime}$, which is the projection of viewpoint $\mathrm{O}$ on the ground plane. This triangle intersects the picture plane along the straight line $\mu(\mathrm{A})$, which gives us the position of (A). Once the picture plane has been collapsed onto the ground plane, as we said before, we do not have viewpoint $O$, but rather its projection $O^{\prime}$ (Figure 4). Thus, if from $\mathrm{O}^{\prime}$ we project the visual ray corresponding to point $\mathrm{A}$, the ray will intersect the base line at point $\mu$. Moving perpendicular to the base line from $\mu$

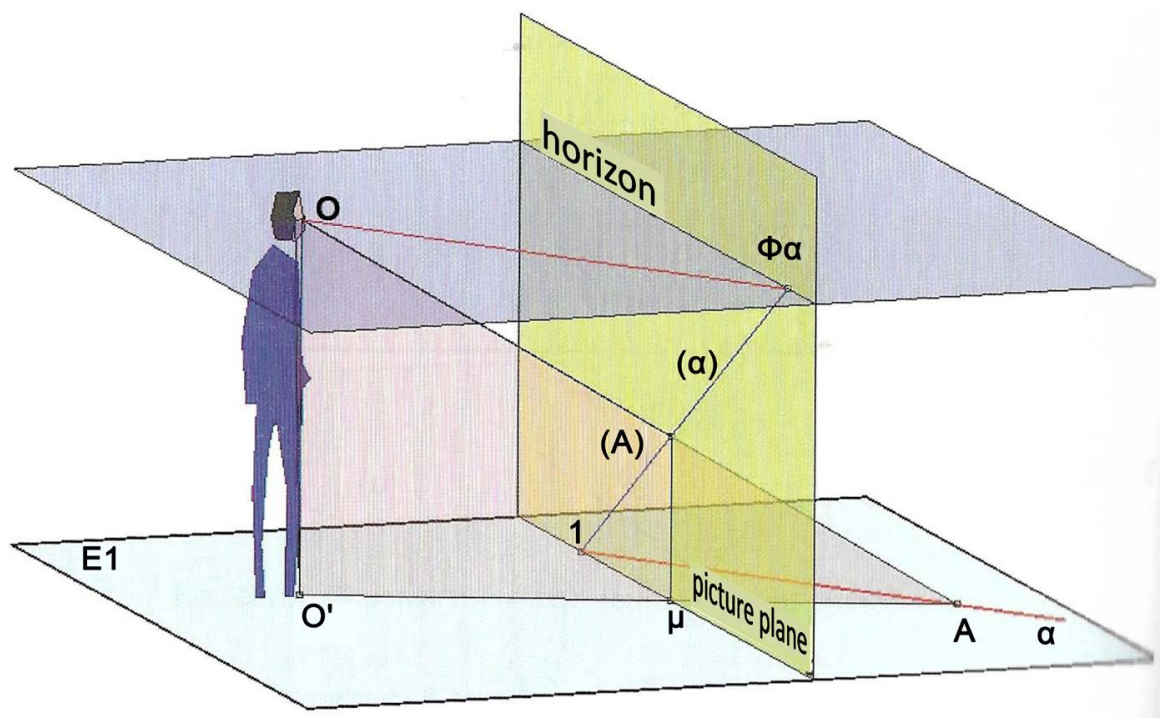

Figure 3. A visual ray meets the picture plane.

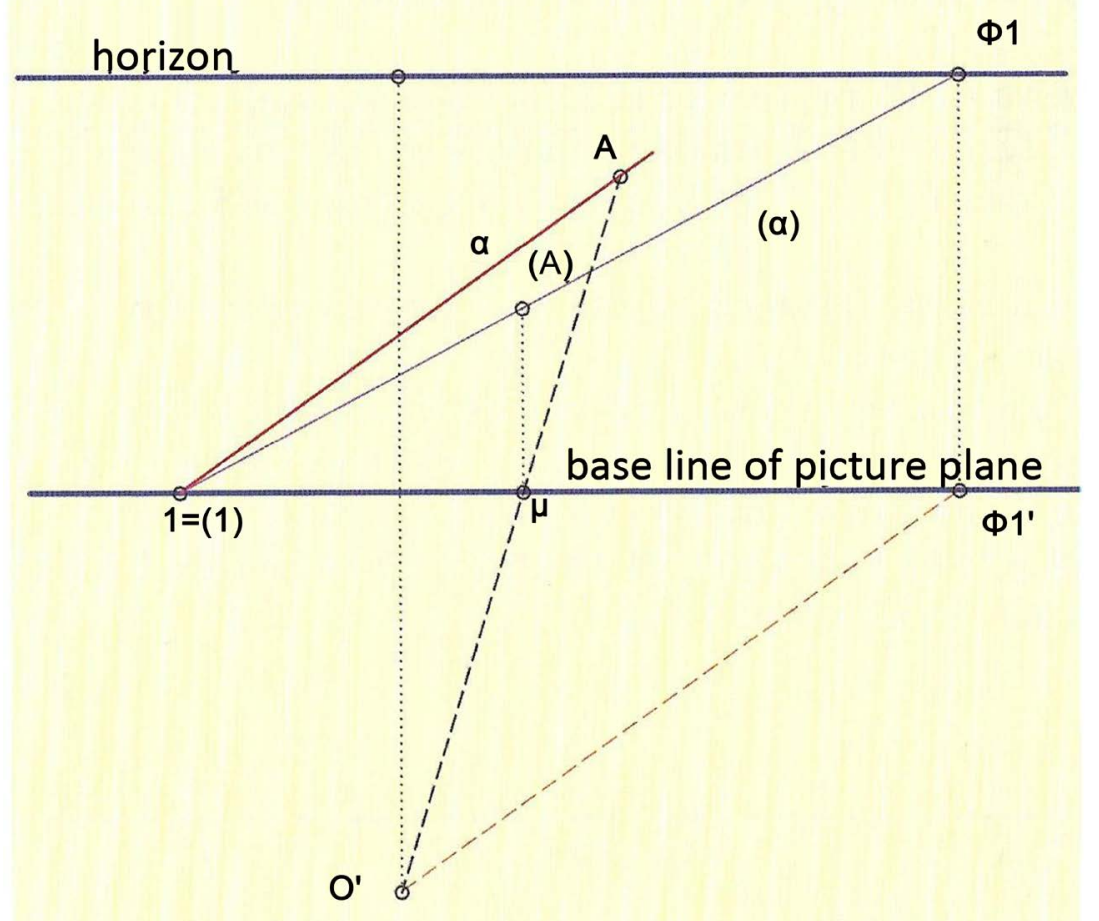

Figure 4. From the 3D axonometric representation to the 2D plane. 
in a straight line, we meet perspective ( $\alpha$ ) of $\alpha$ at point $(\mathrm{A})$, which is the perspective of point $\mathrm{A}$.

\subsection{Perspective Image of Horizontal Line $\alpha$ Located a Distance $Z$ from the Ground Plane}

In Figure 5 we see the perspective of horizontal line $\alpha$ on the picture plane. Line $\alpha$ is the projection of $\alpha$ on the ground plane. Let $\alpha$ 's height be Z. Based on what we have said about finding the perspective of a horizontal line, to find the perspective of $\alpha$ we can proceed as follows:

Line $\alpha$ intersects the picture plane at point $\mathrm{M}$, which is a distance $\mathrm{Z}$ from point 1 of line a' on the picture plane. The vanishing point of $\alpha$ will be $\Phi 1$, which is the same as the vanishing point of $\alpha$, since $\alpha$ and $\alpha$ are parallel in space. Thus, the perspective of $\alpha$ will be line $(\alpha)$, in other words $\Phi 1(M)$. Given that we have the position of $\mathrm{O}^{\prime}$, but not of $\mathrm{O}$, in order to find point $\mathrm{M}$ we can say that from point 1, where the projection $\alpha$ of $\alpha$ intersects the picture plane at its base line, we can extend a line equal to $\alpha$ 's height $Z$, perpendicular to the base line. This perpendicular line ends at $M$, which, if joined with vanishing point $\Phi 1$, gives us the perspective of $\alpha$. We can therefore conclude that the height of a horizontal

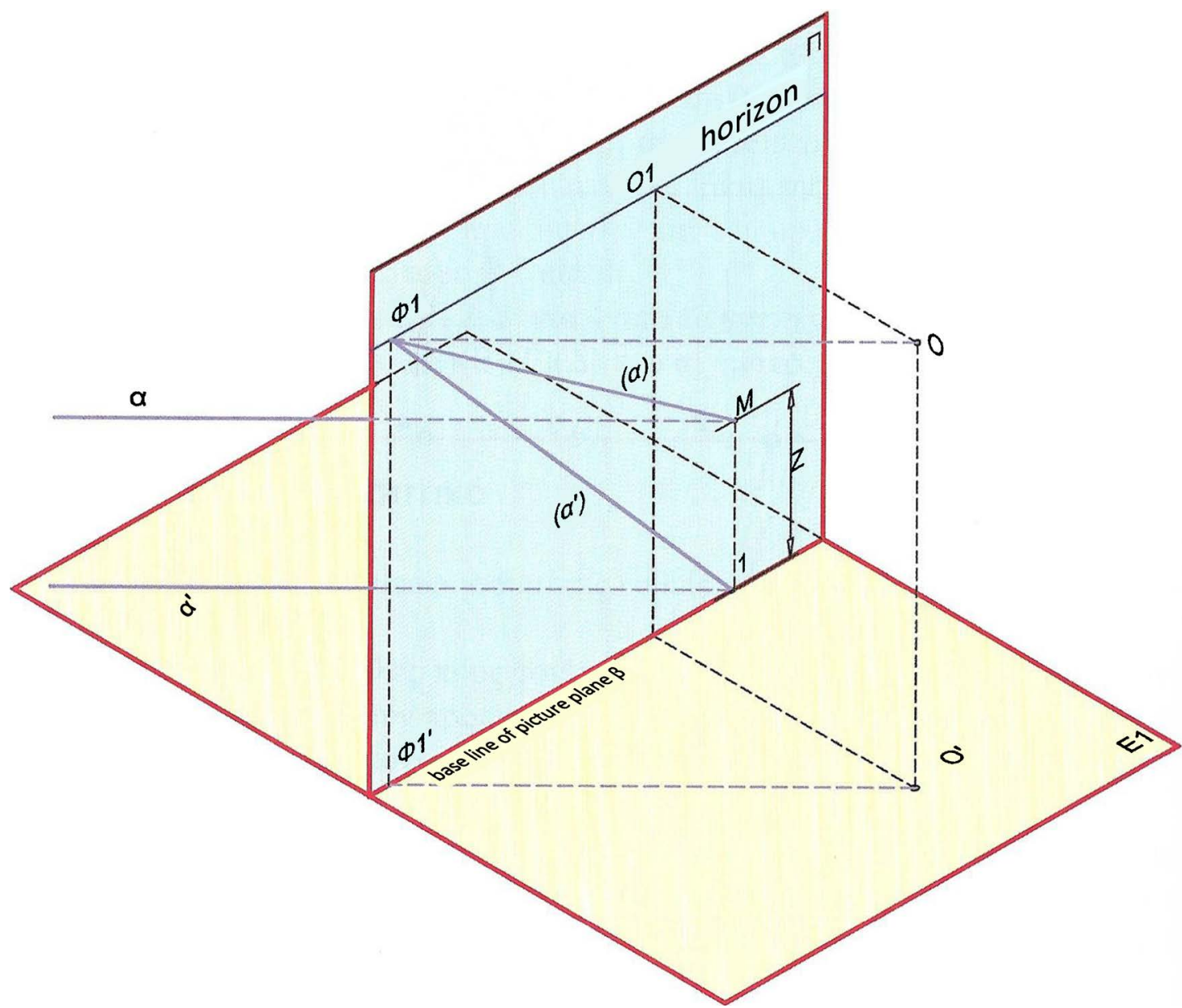

Figure 5. Perspective image of a horizontal line located a distance $\mathrm{Z}$ from the ground plane. 
line, or more commonly its distance from the ground plane, is shown in perspective in its actual size, as the distance from the point where the line's projection intersects the base line and from there in a perpendicular direction to the perspective of the said line. The further we move away from the picture plane and the observer, the smaller the height of the straight line generally becomes in perspective.

\section{Perspective Image of a Frontal Cube. Exploration of the Relationship between Perspective and the Position of an Object in Relation to the Picture Plane}

According to the rules of classical perspective [2] [3], the cube in Figure 6 is projected from point $\mathrm{O}$ onto the picture plane, resulting in its perspective image. Table 1 shows various perspectives of the same cube, placed at different distances from the picture plane. In all cases, the distance between viewpoint $\mathrm{O}$ and the picture plane is fixed. What changes is the cube's position in relation to the picture plane. We observe that when the cube is placed behind the picture plane, all its edges are portrayed smaller than they actually are. When it is placed between the picture plane and the viewpoint, all its edges are portrayed bigger than they really are on the picture plane. When the cube is positioned in such a way that it is intersected by the picture plane, then the part behind the picture plane is portrayed smaller and the part in front of the picture plane bigger. Therefore, in this latter case, the cross-section obtained when the cube is cut by the picture plane, in other words the square belonging on the picture plane, is portrayed in its actual size. This is also made apparent in the last case shown, where the cube has been positioned so that its front face is flush with the picture plane. In perspective, this face is shown in its actual size, whereas the rest of the cube is portrayed smaller.

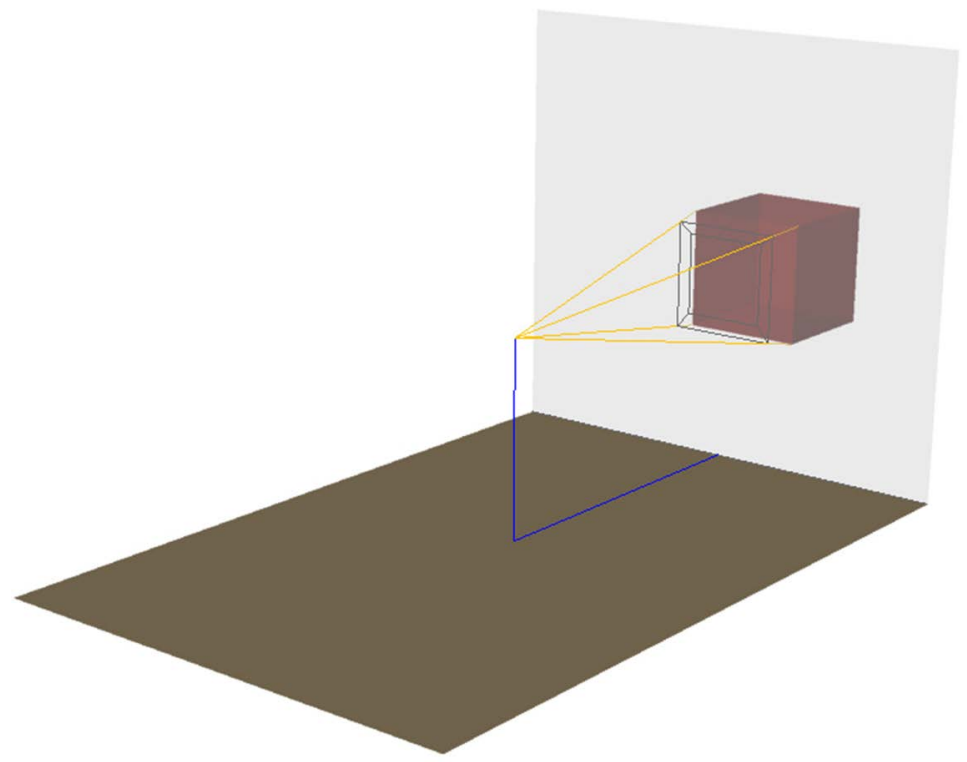

Figure 6. Projection of a frontal cube. 
Table 1. Projection of a cube according to its place in relation to the picture frame.

1

Cube behind picture plane

2

3

Cube intersected by picture plane

4

Cube's front face flush with picture plane

Cube in front of picture plane
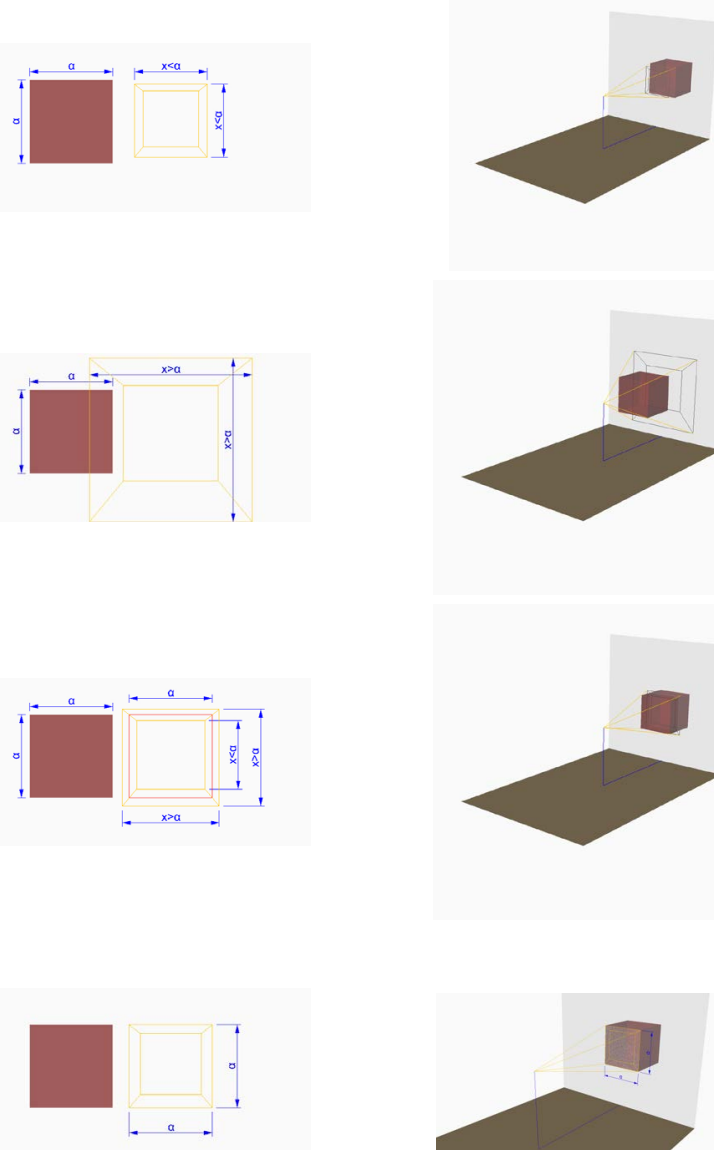
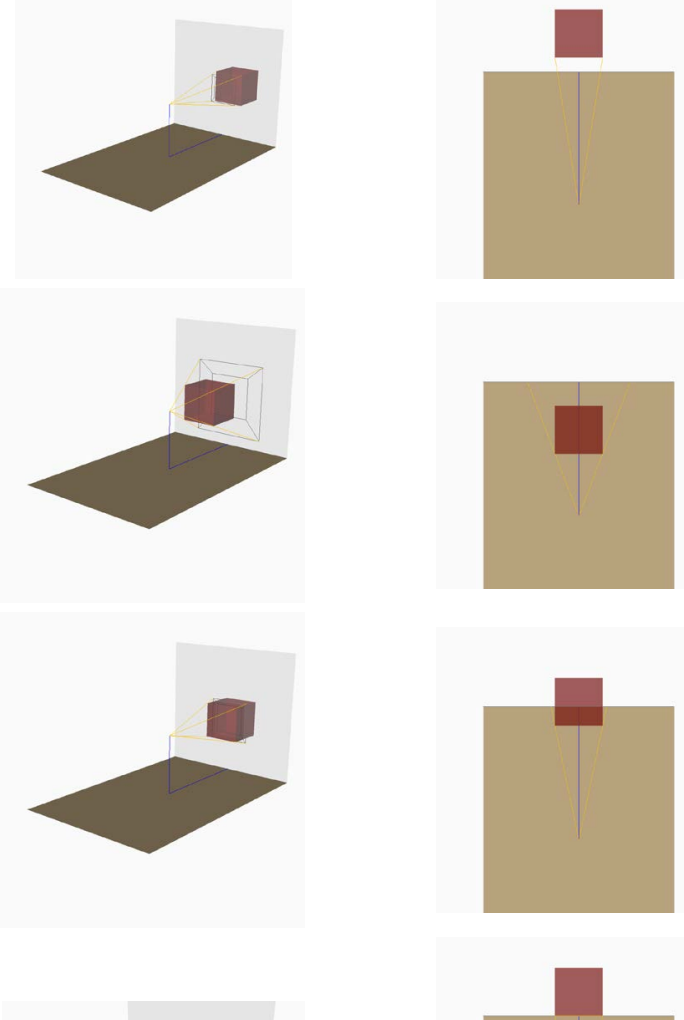

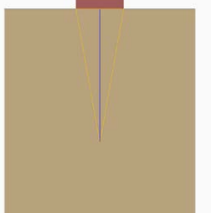

\section{Case Study of Leonardo da Vinci's The Last Supper}

In order to reconstruct a model of the three-dimensional space represented by this painting (Figure 7), we must first assume that the artist has realistically portrayed the interior of a room, and has opened the room up to the viewer by removing the front wall. Let us therefore say that the room's wall, which would have been positioned under the front beam depicted by the artist in the painting's foreground, has been removed. The picture plane of this particular work of art has therefore been positioned where the missing wall would have been. The second assumption concerns the room's ceiling panels. These panels were considered to be perspective drawings of similar, horizontal, square elements, and have been dealt with as such. Following these two assumptions, we will go on to find the picture plane's base line and horizon line and, lastly, the projection of the viewpoint. In other words, we will find at what distance from the work of art, and at what height, the artist positioned the observer's eye. We will then reconstruct the spatial model and draw the perspective of the said model from the same viewpoint as that indicated by the artist. If the vanishing points and relative distances between objects in the resulting model's perspective image coincide with 
the vanishing points and relative distances between the objects in the artist's painting, this will first of all mean that we have correctly determined the artist's desired viewing distance and viewing height. It will also mean that the study's initial assumptions are correct.

To begin with, the painting's qualitative elements, such as colour and shading, are removed, and only its structural elements are redrawn as shown in Figure 8. It was initially observed that the perspectives of the room's horizontal lines all converge toward a single vanishing point, which coincides with Christ's right eye, at the centre of the table (Figure 9). We can thus pinpoint the position of vanishing point $\Phi 1$ of all the horizontal lines in the room. These lines are also perpendicular to the picture plane, in other words perpendicular to the missing front wall (Figure 10). Having determined the vanishing point, we can also tell where the horizon line lies, since the vanishing point is on this line (Figure 11). Furthermore, based on our first assumption, namely that the picture plane is positioned where the wall opened up to the viewer would stand, we can deduce that the base line is located as shown in Figure 11. In Figure 12 we find where the room's ceiling intersects with the plane of the missing wall and we extend the receding lines of the walls' two top borders until they meet the two vertical lines that form the borders of the front wall. The ceiling plane is then divided into

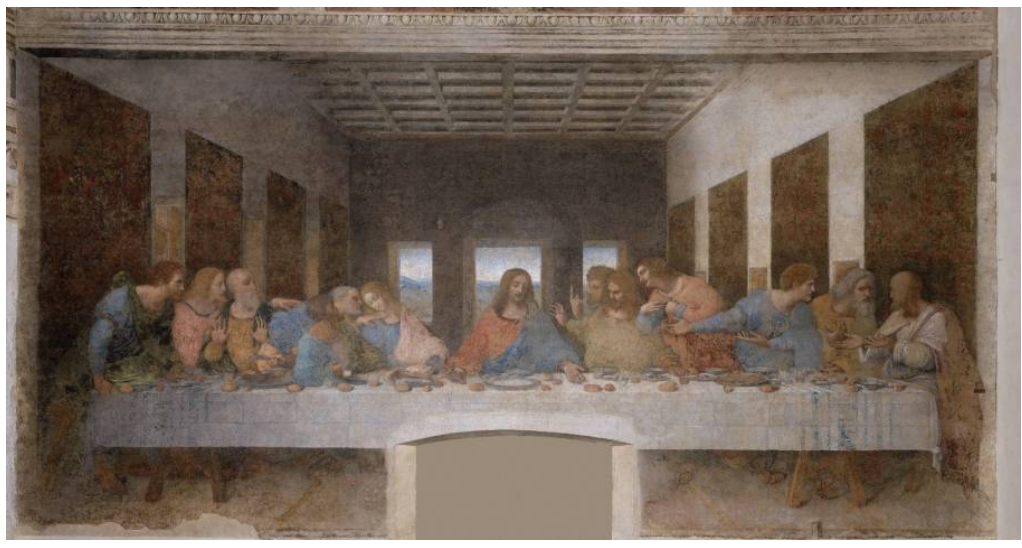

Figure 7. The work of art. https://www.leonardodavinci.net/the-last-supper.jsp

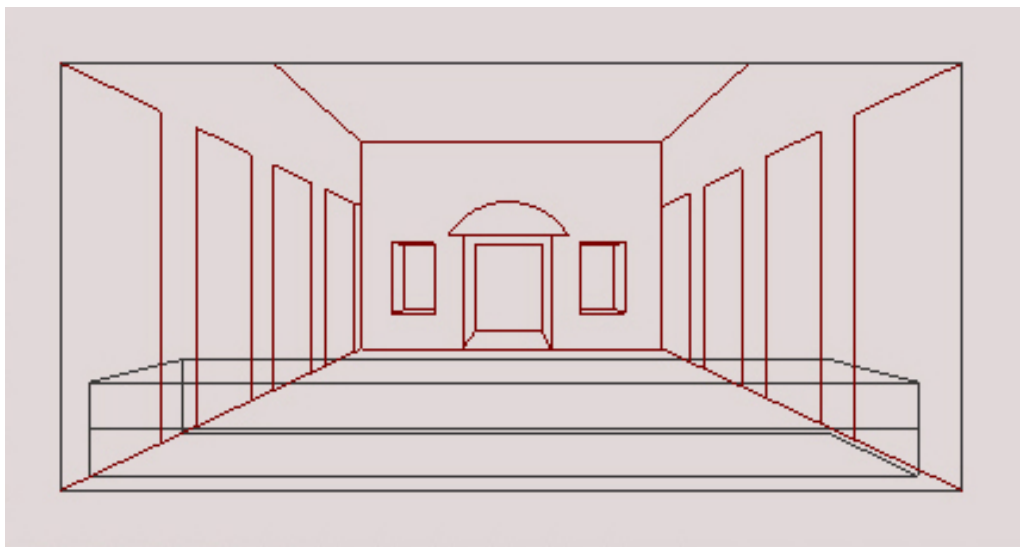

Figure 8. The initial drawing. 


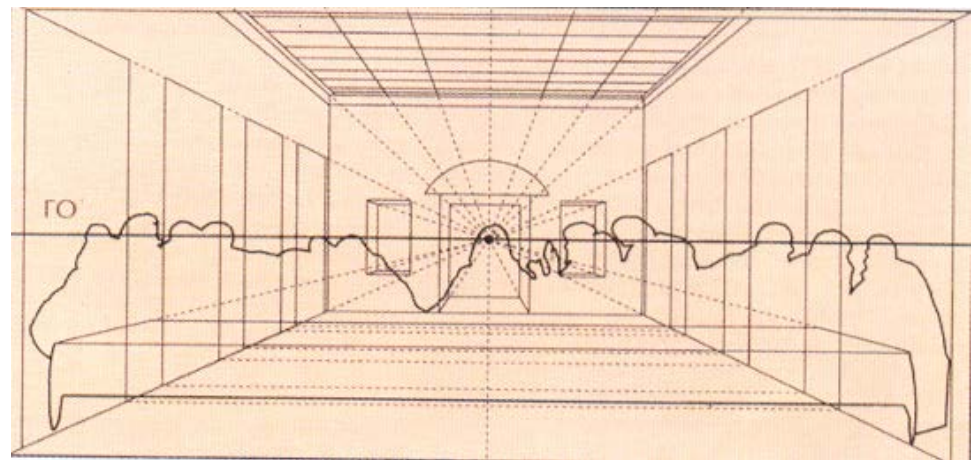

Figure 9. The central point of view is the eye of Jesus.

https://www.leonardodavinci.net/the-last-supper.jsp

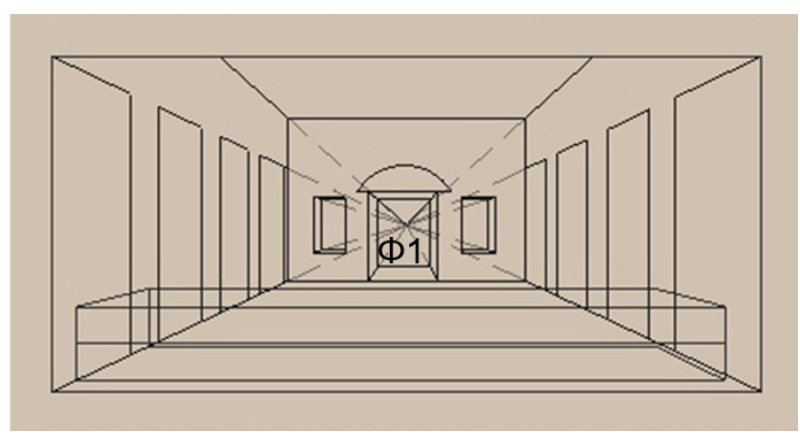

Figure 10. The vanishing point.

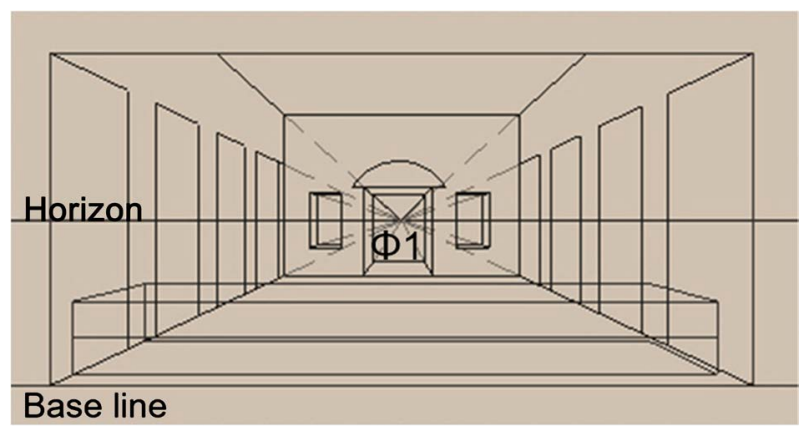

Figure 11. The line of horizon and the base line of picture plane.

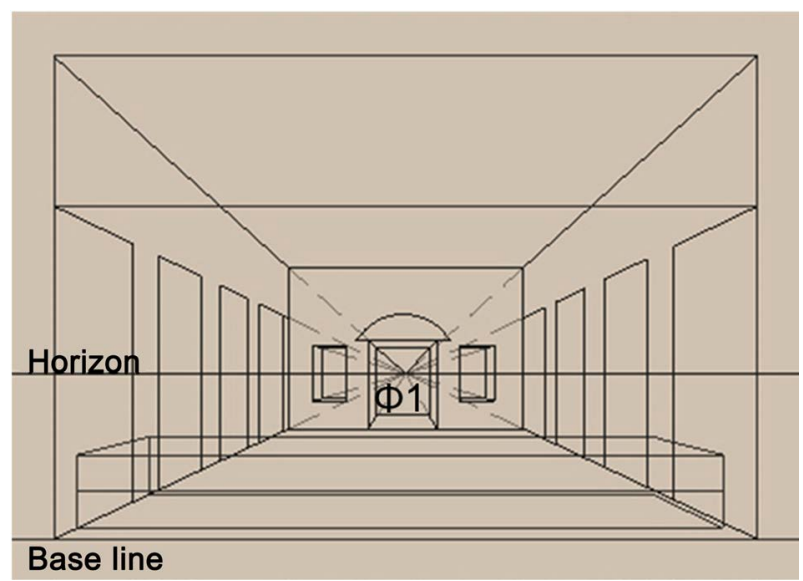

Figure 12. Reconstructing the ceiling of the room. 


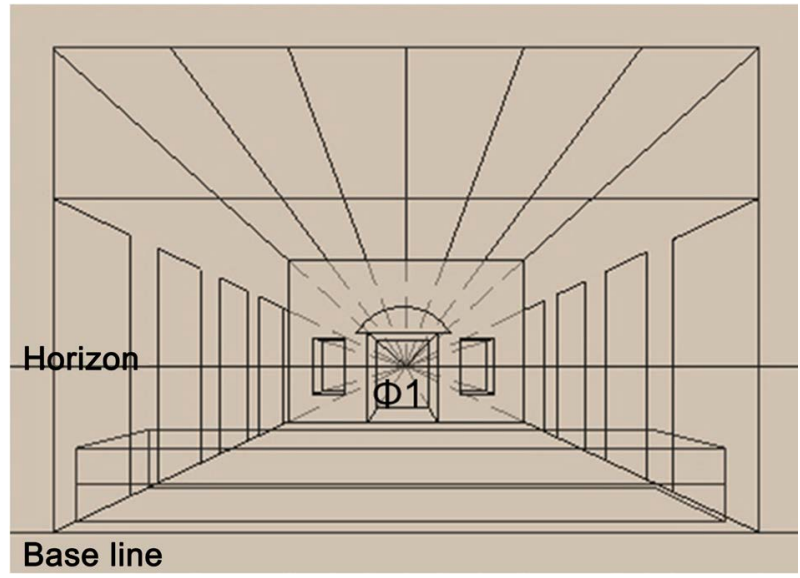

Figure 13. Reconstructing the ceiling of the room.

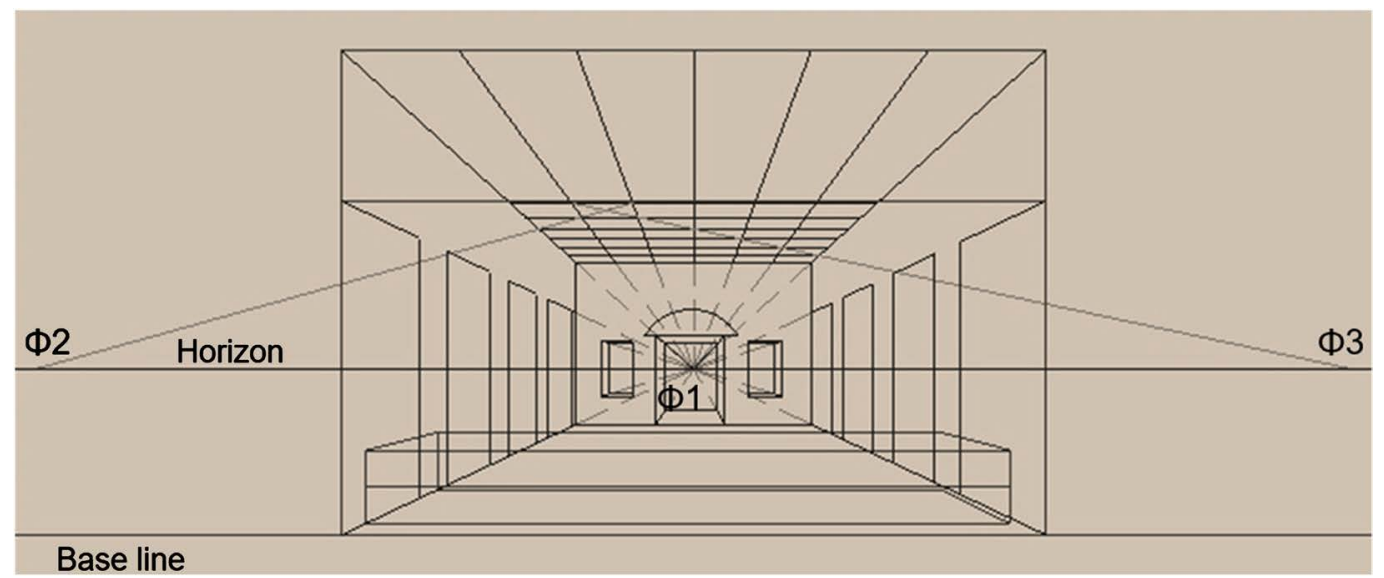

Figure 14. The vanishing points of the diagonals.

equal perspective parts, given our second assumption that the ceiling consists of equal square panels (Figure 13). Figure 14 also shows the horizontal beams, thus providing a perspective of all the squares on the ceiling. Since these squares are horizontal, their diagonals will also be horizontal lines. Consequently, the vanishing points of lines $\Phi 2$ and $\Phi 3$ will also be along the horizon line. The projections of $\Phi 2$ and $\Phi 3$ on the base line will be $\Phi 2^{\prime}$ and $\Phi 3^{\prime}$ (Figure 14/Figure 15). A square's two diagonals form a $90^{\circ}$ angle between them. Consequently, if we had the position of the projection of viewpoint $\mathrm{O}^{\prime}$, to find $\Phi 2^{\prime}$ and $\Phi 3^{\prime}$ we would draw parallel lines from $\mathrm{O}^{\prime}$ towards the two diagonals. These parallel lines would also meet at a $90^{\circ}$ angle. The locus of the points on the plane that meet section $\Phi 2^{\prime} \Phi 3^{\prime}$ at a $90^{\circ}$ angle is a semicircle whose diameter is $\Phi 2^{\prime} \Phi 3^{\prime}$, as shown in Figure 15. However, $\mathrm{O}^{\prime}$ also runs perpendicular to the picture plane's base line from $\Phi 1^{\prime}$. The position of $\mathrm{O}^{\prime}$ is thus the intersection of the two loci, as seen in Figure 16.

\section{Reconstruction of the Spatial Model Representing the Work of Art}

As we continue with our study, to facilitate our drawing, so that the ground plan of the reconstructed space does not overlap its perspective, we will move the base 
line and projections of the vanishing points a bit further up our paper (Figure 17). Figure 18 shows how the general outline of the room's ground plan is reconstructed [1]. The first thing we position on the ground plan is the missing front wall, which, based on the first assumption we made in our study, is in its

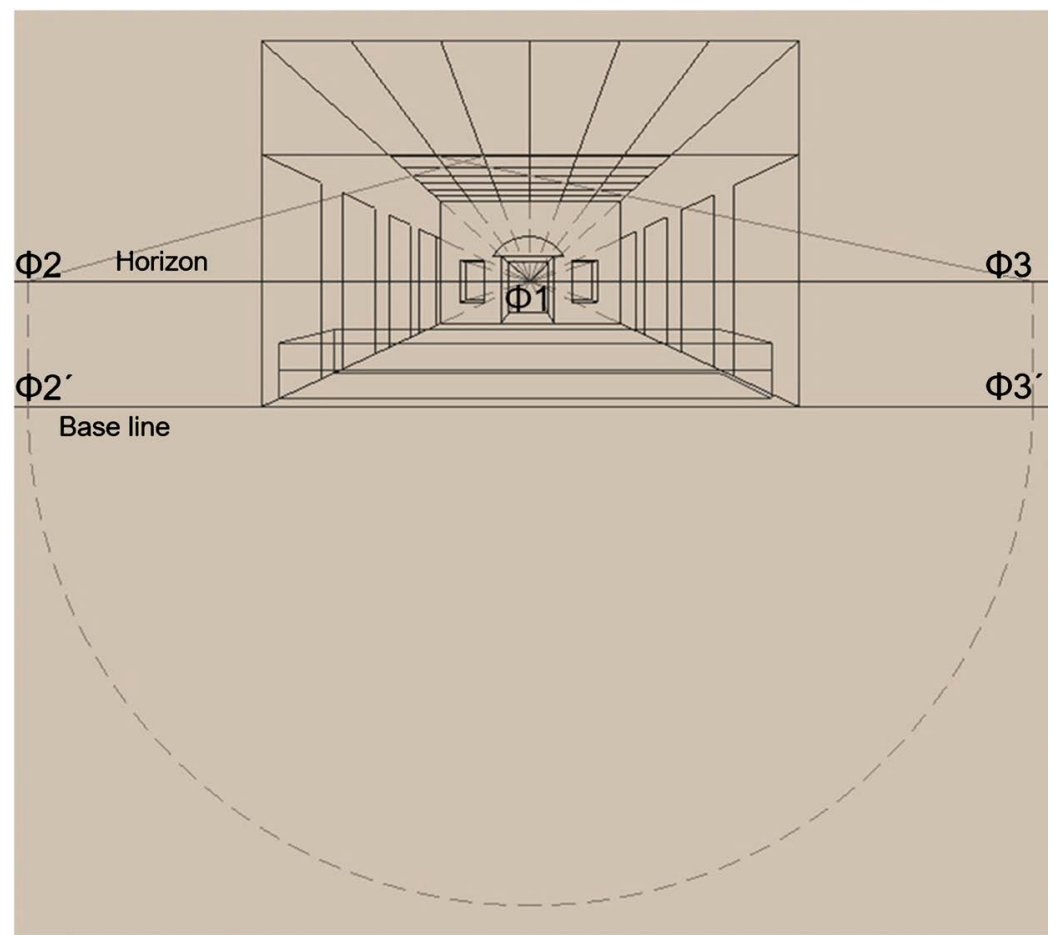

Figure 15. The first loci of the point of view O'.

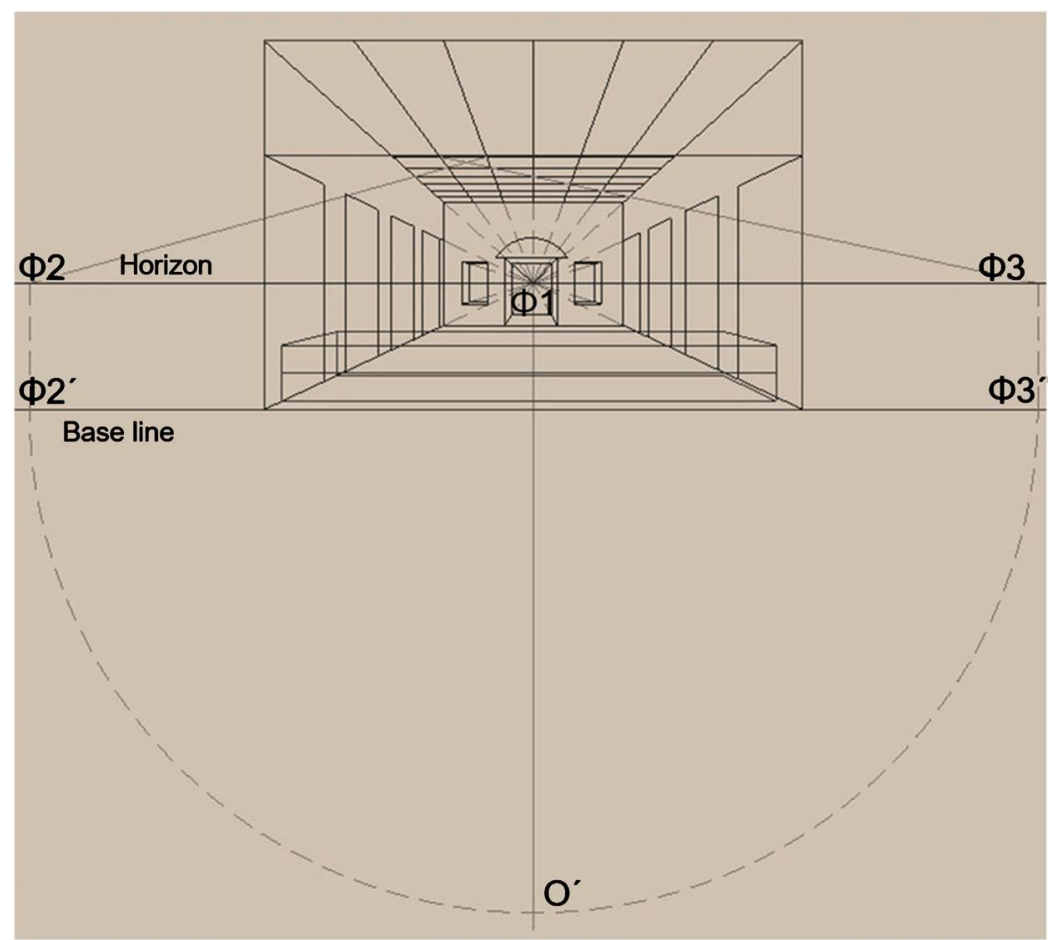

Figure 16. The second loci of the point of view O' and its actual placement. 


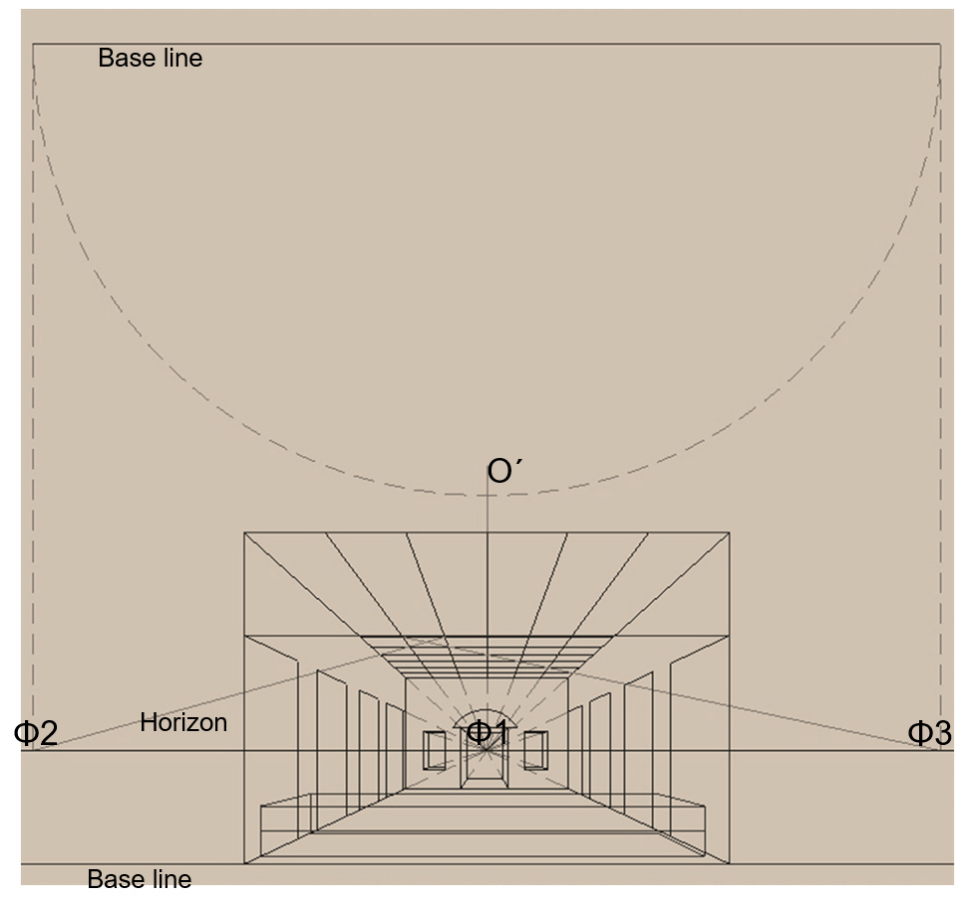

Figure 17. The starting point of the reconstruction of the plan of the room.

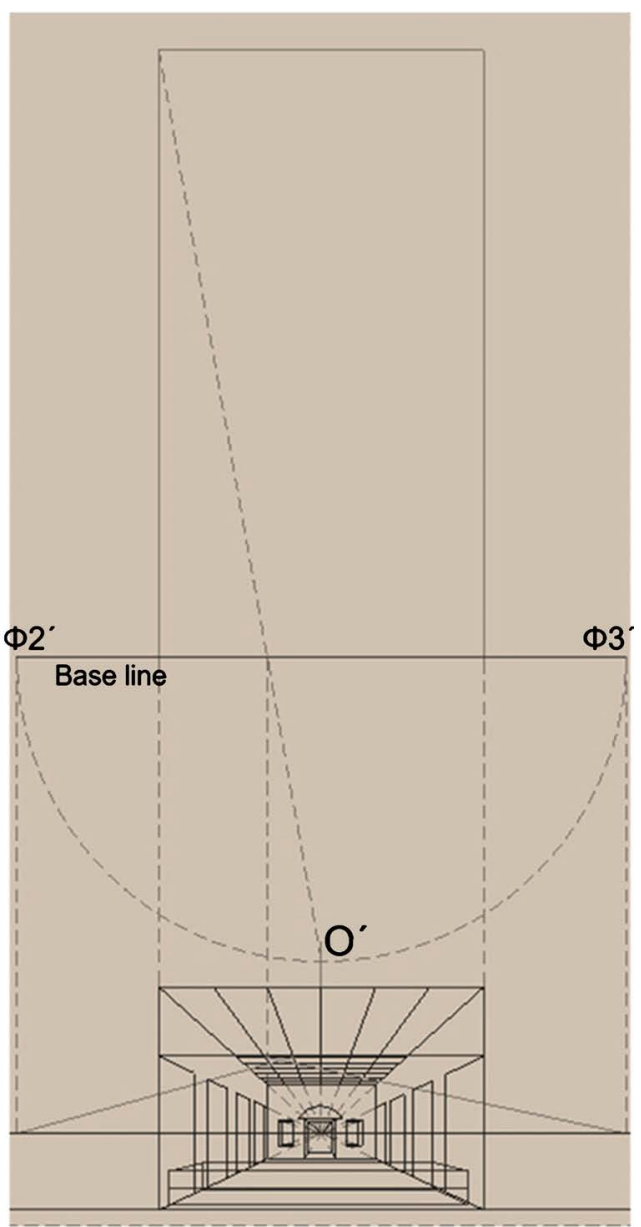

Figure 18. The size of the room in plan. 
actual size since it is located on the picture plane. The perspective drawing allows us to directly deduce the room's width and height, which are depicted in their actual size, as seen in the corresponding case with the cube. We then use the visual ray technique mentioned early on in the article to pinpoint the position of the room's back wall on the ground plan: From the perspective drawing, we project the front wall's left border, as well as the back wall's left border, onto and perpendicular to the base line (Figure 18). The vertical wall will be perpendicular to the base line on the ground plan (since the perspective drawing's vanishing point is $\Phi 1$ ); it will begin at the picture plane and end at the point where the visual ray beginning at $\mathrm{O}^{\prime}$ intersects the perpendicular line. Thus, with the help of the visual rays mentioned before, we can form the outline of the room's ground plan. The positions of the table (Figure 19) and the openings along the walls (Figure 20) are also located on the ground plan in a similar way. The heights of the various elements are measured on the picture plane once their perspective sizes have been projected from $\Phi 1$ onto the plane, where they will be shown in their actual size (Figure 21). The heights of the walls, various openings and the table are found in this way.

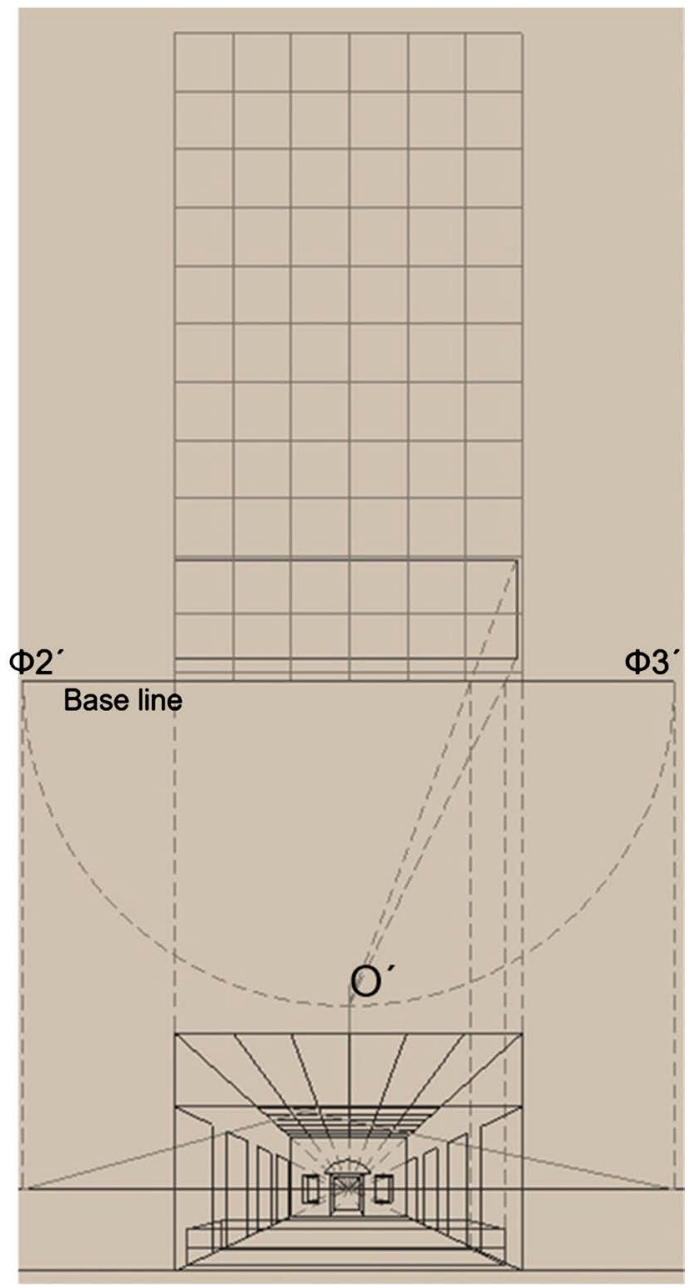

Figure 19. The reconstruction of the ceiling and of the table. 


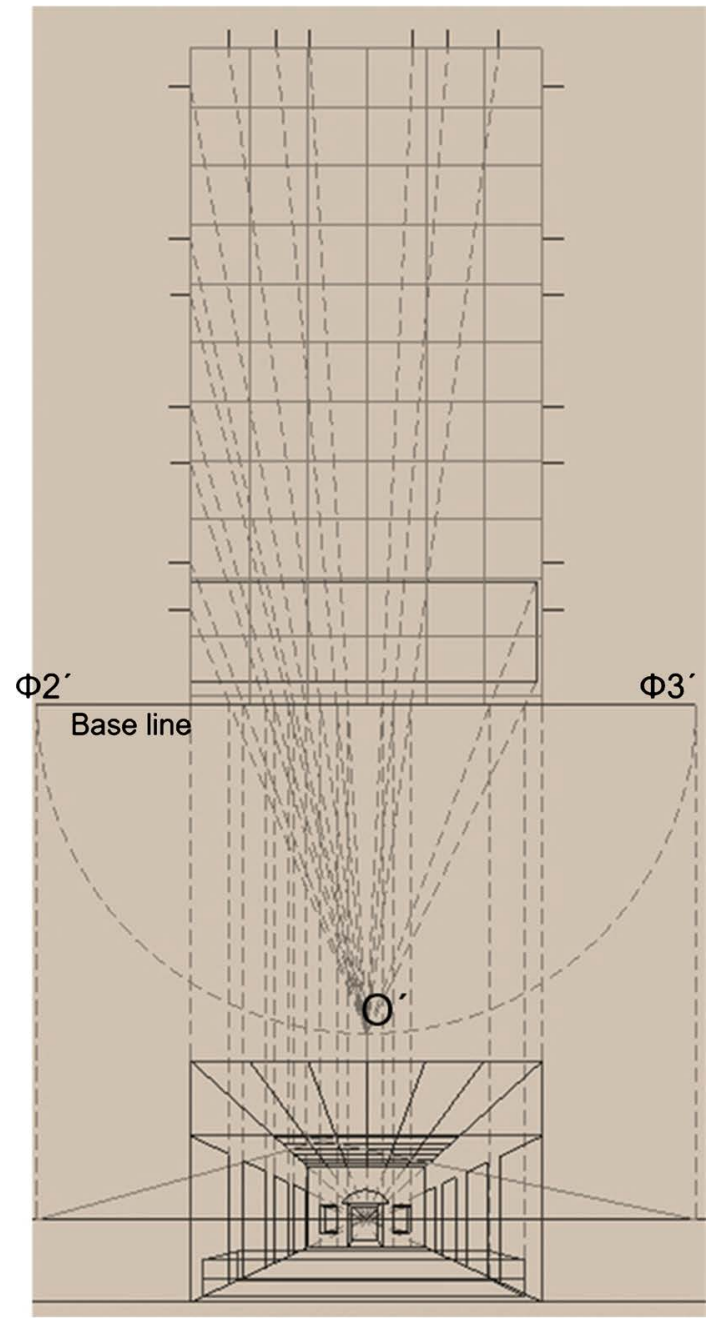

Figure 20. The reconstruction of room openings.

\section{The Results}

Figure 22 shows a three-dimensional model of the room depicted in the work of art, based on the measurements taken in the previous stage of the study. The model was made using the program Rhinoceros. In Figure 22 we can see the viewpoint's position in relation to an abstract version of the painting positioned where the missing wall would be. The height of the viewpoint is equal to the distance between the horizon line and base line, as we noted early on in the study. In this picture we can also see distance O'O, which symbolises the viewpoint's height from the missing wall's lower boundary, which coincides with the picture plane's base line. Therefore, based on the art work's size, which we know in this instance, we can calculate the distance that it should have from the floor of the room in which it is exhibited, so that the correcting viewing height can be achieved. The optimal viewing distance can also be calculated, given that the distances established in this study are dependent on the scale prescribed by the initial image we had in our possession. However, knowing, as we do, the actual dimensions of this work of art, we can deduce the measurements and determine 


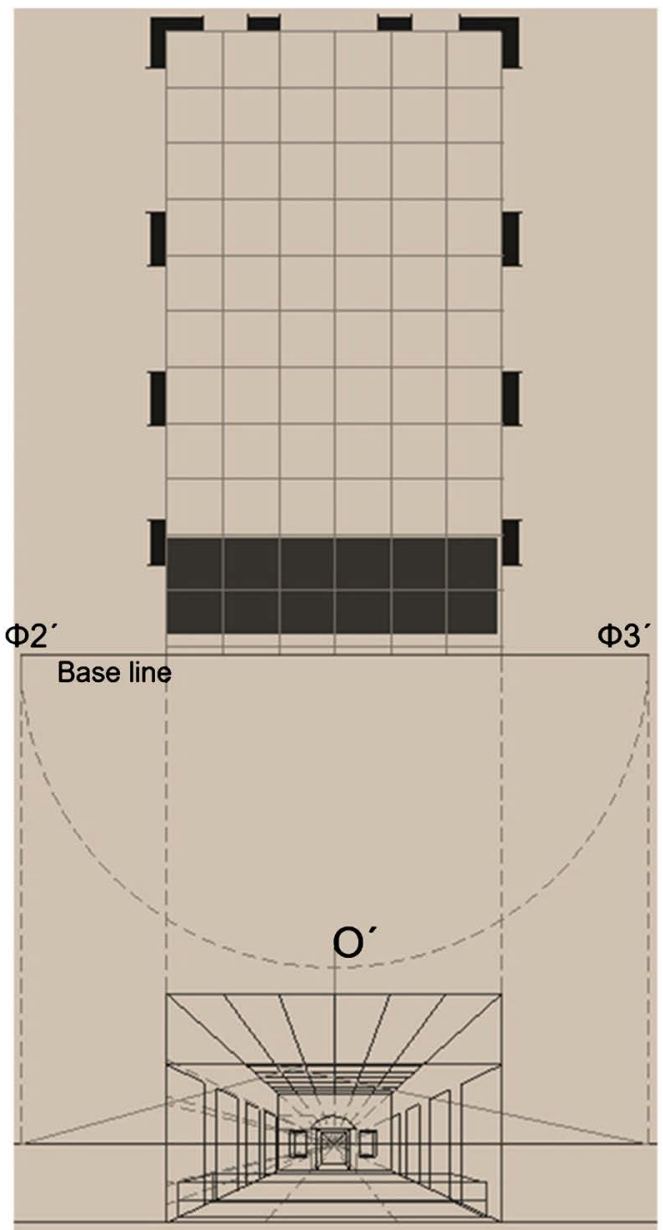

Figure 21. The reconstructed plan.

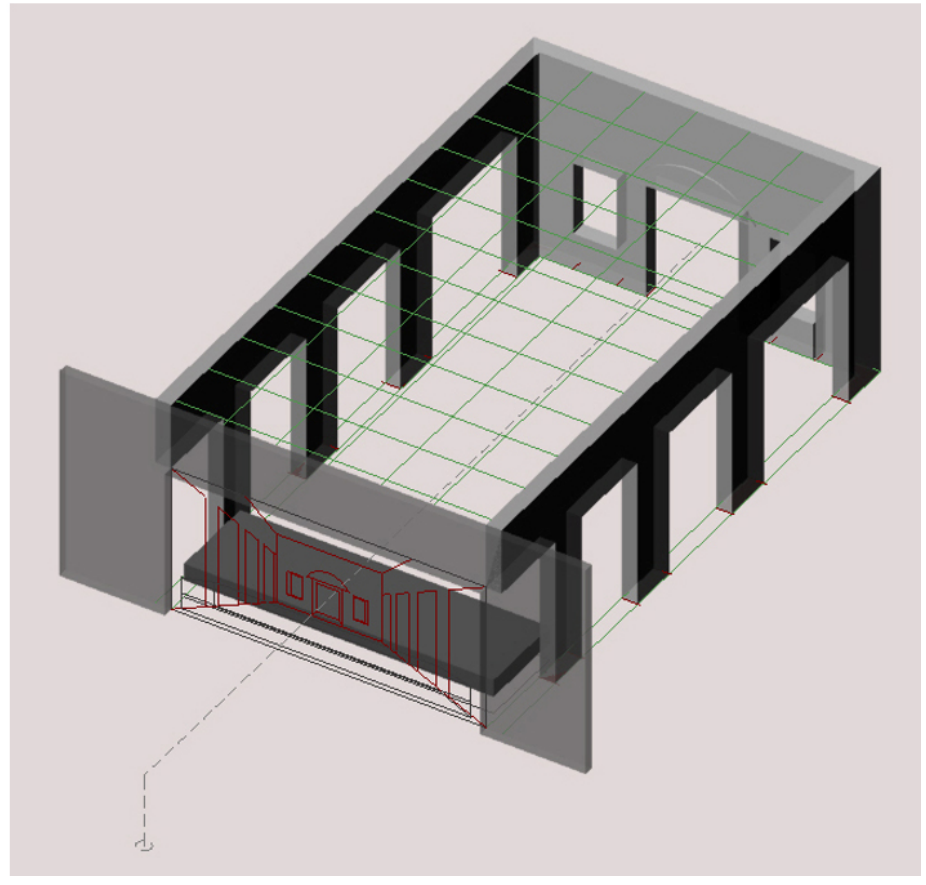

Figure 22. The axonometric vies of the room. 
the real viewing distance and viewing height.

Figure 23 shows the perspective of the spatial model from the station point, as depicted using the program Rhinoceros [4], with the program's camera positioned at the station point and the camera's target point set at point $\Phi 1$. Lastly, in Figure 24 we see the same perspective, with the image of the art work having been positioned where the missing front wall would be, in other words, where the picture plane is, based on the first assumption made in the study. As a result, we observe that the perspective drawing of the room and the artist's painting coincide to a great extent. There is correspondence between both the receding lines and the relative distances between the depicted objects. This means that the initial assumptions made in the study are correct and also that our approach to the room that the artist had before him or imagined when he created this work of art is relatively precise.

This study suggests a geometrical method from 2D to 3D imaginary space [4]. This approach could open a discussion in the field of Art concerning the relation between real and imaginary space that could possibly generate a connection

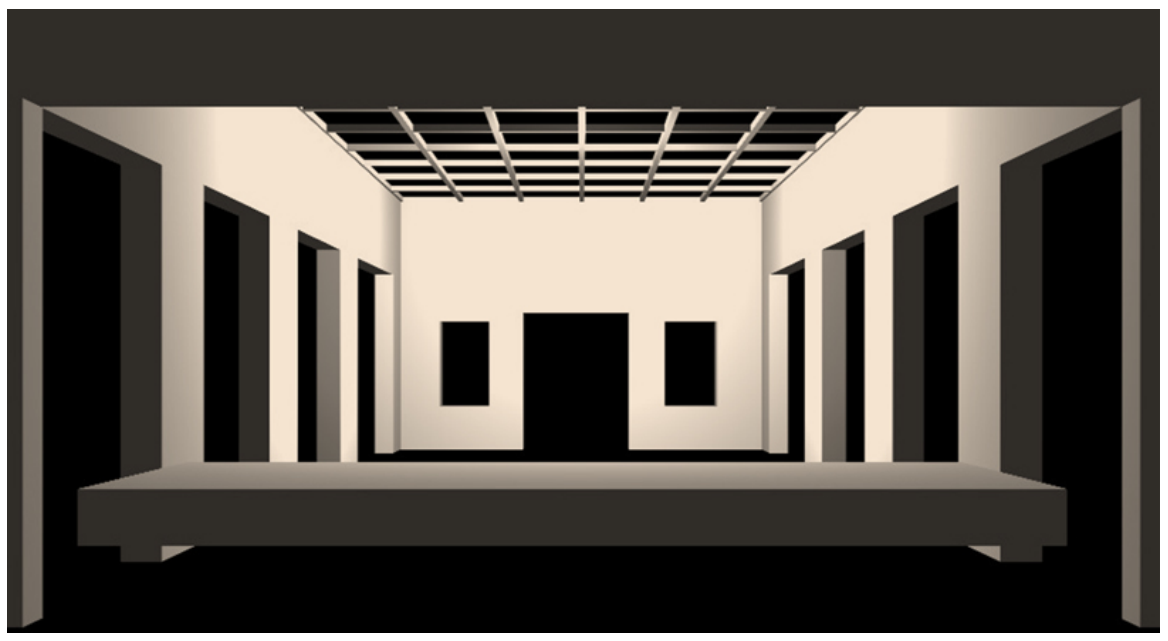

Figure 23. The perspective view of the room from the reconstructed point of view.

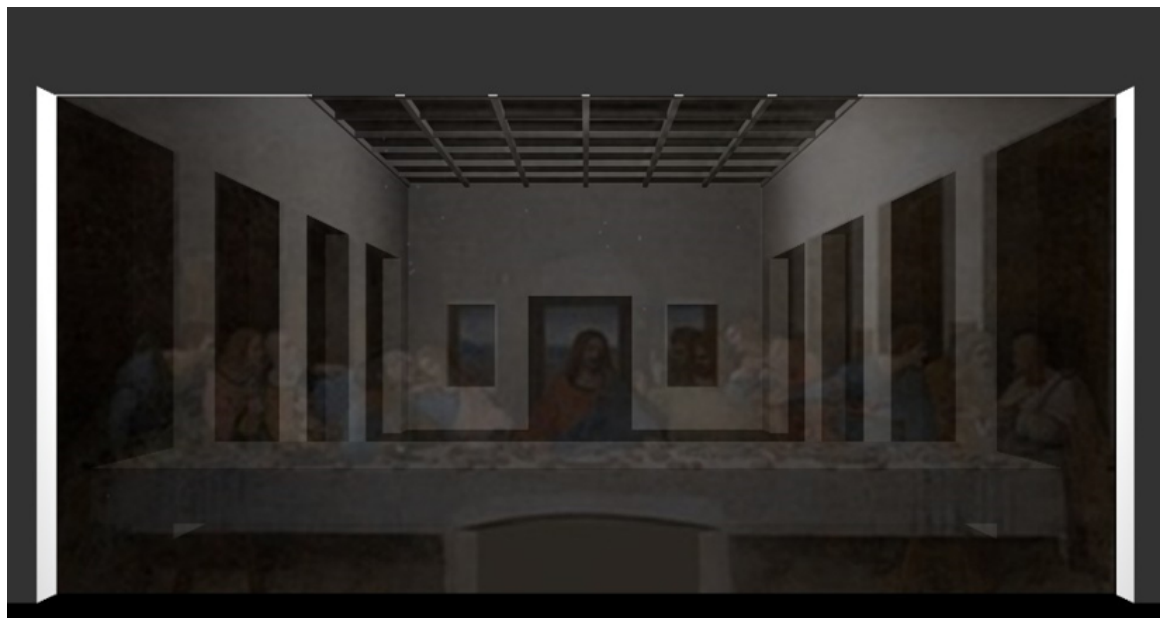

Figure 24. The perspective image and da Vinci's painting coincide. 
between Art and Architecture, using a pure geometrical synthetic tool. After all, geometry has always been a very important base knowledge in the education of architects.

\section{Acknowledgements}

I would like to thank Professor Anthi Maria Kourniati (School of Architecture, National Technical University of Athens, Greece) for her advice on the geometrical methods.

\section{Conflicts of Interest}

The authors declare no conflicts of interest regarding the publication of this paper.

\section{References}

[1] Kourniatis, N. and Kourniati, A.M. (2011) Perspective in Architectural Representation. Tziolas Publications, Thessaloniki.

[2] Kourniati, A.M. (1997) Euclid's Optics and Perspective Representations. Ph.D. Dissertation, National Technical University of Athens, School of Architecture, Athens.

[3] Kourniatis, N. (2013) Space as a Common Concept Assigning Meaning to Geometry and Architecture. From Baroque Projection to Contemporary Parametric Design of a Topological Nature. Ph.D. Dissertation, National Technical University of Athens, School of Architecture, Athens.

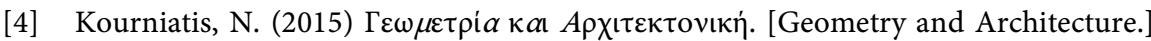
Tziolas Publications, Thessaloniki. 